\title{
Incorporation of ${ }^{35} \mathrm{~S}-\mathrm{Methionine}$ or ${ }^{35} \mathrm{~S}-\mathrm{Cy}$ stine into Polypeptide Protease Inhibitor in the Rabbit Skin with Healing Inflammation (Preliminary Report)
}

\author{
Takeshi Kambara, Tachio Aimoto and Hideo Hayashi \\ Department of Pathology (Prof. H. Hayashi), \\ Kumamoto University Medical School, Kumamoto
}

\begin{abstract}
The specific inhibitor of $\mathrm{SH}$-dependent skin protease responsible for the development of Arthus-type inflammation was isolated from the skin site and highly purified by chromatography. The gradual cessation of the inflammatory process was associated with a local increase of the inhibitor. The inhibitor (polypeptide) behaved as a homogeneous substance in electrophoresis and ultracentrifugation. The constituent amino-acids of the polypeptide were as follows: Cys, Met, Asp, Thr, Ser, Glu, Pro, Ala, Gly, Val, Leu, Ileu, Phe, Lys, His, Arg and HO-pro. The labeled amino-acids ( ${ }^{35} \mathrm{~S}$-methionine or ${ }^{35} \mathrm{~S}$-cystine) given intravenously were incorporated into the inhibitor; approximately 87 per cent of radioactivity in the hydrolysed inhibitor was found in methionine and approximately 13 per cent in cystine. The observations suggested possible synthesis of this inhibitor in the inflamed skin site.
\end{abstract}

In previous studies, ${ }^{1,2}$ we demonstrated that the activity of a certain SHdependent skin protease increased in parallel with the development of Arthustype inflammation in the rabbit skin and that the inactivation of the protease by local increase of a specific inhibitor was followed by a gradual cessation of the inflammatory process. The inhibitor (polypeptide) isolated behaved as a homogeneous substance on electrophoresis and ultracentrifugation. ${ }^{3,4}$ It inactivated papain (EC $3 \cdot 4 \cdot 4 \cdot 10$ ) as well as the Arthus SH-dependent protease (peptide peptidohydrolase) but had no effect on trypsin (EC 3.4.4.4) or a-chymotrypsin (EC 3.4. 4.5). The present paper deals with in vivo incorporation of ${ }^{35} \mathrm{~S}$-methionine or ${ }^{35} \mathrm{~S}$ cystine into the polypeptide inhibitor.

In ten sites in an area of $12 \times 15 \mathrm{~cm}$ on the clipped flanks of bovine serumsensitized rabbits $(2-2.5 \mathrm{~kg})$, Arthus-type inflammation was equally induced by intradermal injections of the antigen. ${ }^{1}$ At 24 or 48 hours after the antigen, L- ${ }^{35} \mathrm{~S}$ methionine $(0.23 \mathrm{mc} / \mathrm{kg}$ body-weight, Radiochemical Centre, Amersham, Bucks, Great Britain) or $\mathrm{L}^{-35} \mathrm{~S}$-cystine $(0.23 \mathrm{mc} / \mathrm{kg}$ bodyweight, Schwarz Bioresearch, Inc., Orengeburg, N.Y.) was injected intravenously.

Received for publication, December 8, 1967.

This work was supported by a grant from the Abbott Laboratories, Illinois, U.S.A. 
Two or four days after intravenous injections of labeled amino-acids, the inhibitor was isolated by the method previously described $;^{3}$ its specific activity was approximately 1,700 . The radioactivity of the 'purified' inhibitor was determined, 5 and the results obtained are summarized in Table 1.

TABLE 1. Incorporation of ${ }^{35} \mathrm{~S}$-methionine or ${ }^{35} \mathrm{~S}$-cystine into protease inhibitor at different times after injections of labeled amino-acids (c.p.m./mg)

\begin{tabular}{l|c|c}
\hline \multirow{2}{*}{ Labeled amino-acids } & \multicolumn{2}{|c}{ Radioactivity } \\
\cline { 2 - 3 } L. ${ }^{35}$ S-methionine & 2 day & 4 day \\
L. ${ }^{35}$ S-cystine & 303 & $250^{*}$ \\
\end{tabular}

* The values recorded represent the averages of several determinations.

The hydrolysate of the inhibitor was radiochromatographed. ${ }^{6}$ The chromatogram of the hydrolysates seemed to show radioactivity essentially in the methionine and cystine positions (Fig. 1). Methionine given intravenously was in part transformed into cystine and incorporated into the inhibitor. ${ }^{7}$ The appearance of methionine oxides (methionine sulfoxide and methionine sulfone) and cysteic acid in the radiochromatograph seemed to be due to the oxidation of methionine and cystine during hydrolysis of the inhibitor with hydrogen chloride.

The distribution of radioactivity in the hydrolysed inhibitor is shown in Table 2. In the inhibitor, approximately 87 per cent of radioactivity was found in methionine and approximately 13 per cent in cystine.

A qualitative analysis by automatic amino-acid analyser of constituent aminoacids of the inhibitor after hydrolysis clearly revealed the presence of Cys, Met, Asp, Thr, Ser, Glu, Pro, Ala, Gly, Val, Leu, Ileu, Phe, Lys, His, Arg and HOpro. The inhibitor itself also showed a positive staining with platinum chloride or with nitroprusside followed by sodium cyanide. ${ }^{8}$ The observations just presented indicate that ${ }^{35} \mathrm{~S}$-methionine or ${ }^{35} \mathrm{~S}$-cystine is incorporated into a specific protease inhibitor and that they are actually constituent amino-acids of this polypeptide. Furthermore, the observations would suggest a possibility that the local increase of this polypeptide in parallel with the subsiding inflammatory process may be associated with the synthesis of this substance in the inflamed skin site. This possibility seems very important for the proteolytic mechanism in inflammation.

TABLE 2. Distribution of radioactivity in methionine and cystine residues in protease inhibitor after injection of ${ }^{35} S$-methionine (c.p.m./mg)

\begin{tabular}{c|c|c}
\hline \multicolumn{3}{c}{ Radioactivity } \\
\hline Methionine & Methionine oxides & Cystine, cysteic acid \\
\hline $56^{*}$ & 240 & 44
\end{tabular}

* The values recorded are the averages of several determinations. 

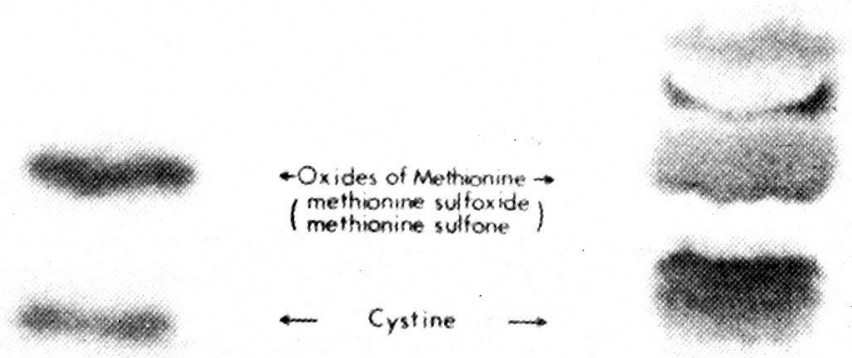

Autoradiogram

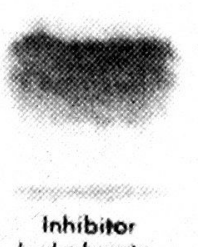

hydrolyzate

Fig. 1. Radiochromatograph of hydrolysate of protease inhibitor showing incorporation of ${ }^{35} \mathrm{~S}$-methionine in the protease inhibitor. The inhibitor was hydrolysed with $6 \mathrm{~N}$ HCI at $105^{\circ} \mathrm{C}$ for 24 hours and chromatographed with mixture of n-butanol-acetic acid-deionized water. Stained with ninhydrin (right).

\section{Acknowledgment}

We are indebted to Dr. T. Murata and Dr. S. Eto, Kumamoto University Faculty of Pharmaceutical Sciences for their advices.

\section{References}

1) Hayashi, H., Udaka, K., Miyoshi, H. \& Kudo, S. Further study of correlative behavior between specific protease and its inhibitor in cutaneous Arthus reactions. Lab. Invest., 1965, 14, 665-673.

2) Hayashi, H., Miyoshi, H., Nitta, R. \& Udaka, K. Proteolytic mechanism in recurrence of Arthus-type inflammation by thiol compounds. Brit. J. exp. Path., 1962, 43, 564 573.

3) Udaka, K. \& Hayashi, H. Further purification of a protease inhibitor from rabbit skin with healing inflammation. Biochim. biophys. Acta (Amst.), 1965, 97, 25】-26!, 
4) Udaka, K. \& Hayashi, H. Molecular-weight determination of a protease inhibitor from rabbit skin with healing inflammation. Biochim. biophys. Acta (Amst.), 1965, 104, 600-603.

5) Werbin, H., Chaikoff, I.L. \& Imada, M.R. Rapid sensitive method for determining $\mathbf{H}^{3}$. water in body fluids by liquid scintillation spectrometry. Proc. Soc. exp. Biol. Med. (N.Y.), 1959, 102, 8-12.

6) Sargent, J.R. \& Campbell, P.N. The sequential synthesis of the polypeptide chain of serum albumin by the microsome fraction of rat liver. Biochem. $J ., 1965,96$, 134-146.

7) Morenkova, S.A. Incorporation of ${ }^{95}$ S-methionine into insulin. Nature, 1966, 209, 917.

8) Toennies, G. \& Kolb, J.J. Techniques and reagents for paper-chromatography. Anal. Chem., 1951, 23, 823-826. 